\title{
Coexistence of hereditary angioedema in a case of familial Mediterranean fever with partial response to colchicine
}

\author{
SEMIHA ERDEM BAHCECI, FERAH GENEL, NESRIN GULEZ, HIKMET T. NACAROGLU
}

Paediatric Allergy and Immunology Department, Dr. Behçet Uz Children's Training and Research Hospital, İzmir, Turkey

\begin{abstract}
Hereditary angioedema (HAE) is a very rare and potentially life-threatening genetic disease characterised by episodes of edema in various parts of the body, including the extremities, face, and airway. The disease is usually associated with attacks of abdominal pain. On the other hand, familial Mediterranean fever $(F M F)$ is an inherited condition characterised by recurrent episodes of painful inflammation in the abdomen, chest, or joints. In this report, we present a child with FMF and undiagnosed HAE, which made him a partial responder to colchicine treatment. Consequently, HAE must be considered in differential diagnosis of cases in which a partial response is obtained from FMF treatment, particularly in countries where FMF is frequently encountered, because early diagnosis of HAE can facilitate prevention of life-threatening complications, such as upper airway obstruction. To our knowledge, our patient is the first patient reported in the literature with the diagnosis of HAE and FMF together.
\end{abstract}

Key words: colchicine, familial Mediterranean fever, hereditary angioedema.

(Centr Eur J Immunol 2015; 40 (1): 115-116)

\section{Introduction}

Hereditary angioedema (HAE) is characterised by recurrent angioedema in skin and mucosa, abdominal pain, nausea, vomiting, diarrhoea, and laryngeal edema; and it is a disease occurring with autosomal mutations in the $\mathrm{C} 1$ inhibitor gene in $25 \%$ of cases, and it is autosomal dominant in $75 \%$ of cases. Almost all patients with HAE have recurrent abdominal pain [1-5]. Familial Mediterranean fever (FMF) is, however, an autoinflammatory, autosomal recessive disorder in which recurrent abdominal pain attacks can be observed in $90 \%$ of cases and joint findings can be seen in $75 \%$ [6]. Hereditary angioedema must be considered in differential diagnosis of cases in which a partial response is obtained from FMF treatment, particularly in countries where FMF is frequently encountered. To our knowledge, our patient is the first reported in the literature with the diagnosis of HAE and FMF together.

\section{Case report}

A 15-year-old girl was referred to us with abdominal pain attacks and swelling in her hands. From the medical history we learnt that abdominal pain attacks proceeding for 9-10 years, sometimes accompanied by fever, occurred three times in a month and got better in 2-3 days. Swelling in her feet had developed approximately 3 years ago and had continued for 2-3 days and then recovered. Owing to R202Q homozygote mutation determined in the $M E F V$ gene, colchicine treatment had been initiated. With colchicine treatment, the complaints of the patient had reduced partially. Because of the family history of HAE in her mother and aunts, recurrent swelling attacks in her extremities, and partial response to colchicine treatment, we evaluated our patient for HAE. C4: $6 \mathrm{mg} / \mathrm{dl}$ (reference value: $10-40 \mathrm{mg} / \mathrm{dl}$ ) and $\mathrm{C} 1$ inhibitor level: $0.05 \mathrm{~g} / \mathrm{l}$ (reference value: $0.14-0.35 \mathrm{~g} / \mathrm{l}$ ) were determined low, as shown above. The patient was diagnosed with hereditary angioedema type 1 , and prophylactic treatment was initiated. The attacks apparently decreased.

\section{Discussion}

Life-threatening airway edema, vomiting, nausea, diarrhea, pain syndromes depending on submucosal oedema, and recurrent edema attacks with no itching can be seen in patients with HAE. Hereditary angioedema is seen in one in 50,000 of the population and no ethnic group difference is determined [2]. According to data related to mutation in C1 inhibitor gene (SERPING1) localised on the $11^{\text {th }}$ chromosome (11q12-q13.1), more than 200 distinct mutations were reported in patients with HAE. Classical HAE has three types: type $1(85 \%)$, type $2(15 \%)$, and type 3 . The

Correspondence: Hikmet Tekin Nacaroglu, Department of Paediatric Allergy, Dr Behçet Uz Children's Hospital, 35220 İzmir,

Turkey, e-mail: tekin212@gmail.com 
clinical tables of both HAE types are indistinguishable from each other but they arise with different mutations [4, 7]. While there is a quantitative and functional reduction in C1INH in type 1 , in type $2 \mathrm{C} 1 \mathrm{INH}$ levels are normal or high but they are dysfunctional. Studies demonstrated that the main mediator increasing the vascular disorders in HAE attacks was bradykinin [4]. Type 3 HAE is characterised by normal cases with antigenic and functional C1INH. Type 3 HAE was named as angioedema related to oestrogen or dependent on oestrogen when it was firstly described. Although the probable mediator causing the formation of this form is not known exactly, it is thought that coagulation factor 12 (Hageman factor) mutation determined in patients and in some of their relatives leads to increased bradykinin production $[4,8]$. Hereditary angioedema is characterised by recurrent localised subcutaneous or submucosal edema lasting 2-5 days. The edema can be anywhere in the body. Skin, upper airway, and gastrointestinal system are the most commonly involved organs. The clinical table can be varied; it is reported that there can be asymptomatic cases, and patients with life-threatening attacks can be observed as well. Recurrent abdominal pains caused by temporary intestinal obstruction due to mucosal edema are observed in almost all patients [4]. Recurrent abdominal pains in $90 \%$ and joint findings in $75 \%$ of patients with FMF can be seen [6]. It is a common situation that patients with HAE owing to gastrointestinal angioedema and cases with FMF because of abdominal pains based on serositis are exposed to unnecessary surgical interventions. The onset age for both diseases is uncertain. It can begin earlier than the first year. The symptoms commenced at 2-3 years of age in patients with HAE aggravate nearly at puberty and they continue for life with varying severity. Patients without any treatment undergo attacks every 7-14 days. Laryngeal attacks are not generally seen before 3 years of age, and they tend to begin after other symptoms $[2,6,9]$. They show similarity with FMF due to recurrent abdominal pains and joint swellings. Colchicine used in the treatment of patients with FMF responds entirely in $70 \%$ and partially in $15 \%$ of patients. There are still $5-10 \%$ of non-responders to colchicine $[10,11]$. Both diseases should be taken into consideration in differential diagnosis in countries where FMF is frequently seen, as in our country. In the presented case, both diseases were determined together and treatments and attacks can be taken under control aimed at both illnesses. Partial response to colchicine treatment and positive family history must alert doctors to HAE in differential diagnosis. It was reported that MEFV has a relation with FMF and PAPA (pyogenic arthritis, pyoderma gangrenosum, and acne) syndrome, cryopyrin-associated periodical syndrome (CAPS); Behçet's disease, Henoch Schönlein purpura, polyarteritis nodosa, PFAPA (periodic fever, aphthous stomatitis, pharyngitis, cervical adenitis), ankylosing spondylitis, multiple sclerosis, and inflammatory bowel disease. An interesting notified relation is the connection between fibromyalgia and MEFV R202Q mutation [11, 12]. No relation has been reported between HAE and FMF so far. According to our data, this was the only case in which the two diseases were observed together. Patients with FMF and HAE whose symptoms were not taken under control, as in our case, must be examined carefully in terms of this aspect.

\section{Conclusions}

In our case FMF and HAE type 1 were detected together, and the clinical findings of both diseases improved with the treatment. Since the clinical findings of these diseases are similar to each other, every patient must be evaluated carefully in terms of both diseases. Our case reveals the importance of additional diseases that can accompany the existing one, and therefore patients who respond to treatment partially should be assessed attentively before a treatment alteration decision.

\section{The authors declare no conflict of interest.}

\section{References}

1. Temino VM, Peebles RS Jr. (2008): The spectrum and treatment of angioedema. Am J Med 121: 282-286.

2. Bowen T, Cicardi M, Farkas H, et al. (2010): 2010 International consensus algorithm for the diagnosis, therapy and management of hereditary angioedema. Allergy Asthma Clin Immunol 6: 24.

3. Busse PJ (2011): Angioedema: differential diagnosis and treatment. Allergy Asthma Proc 32 (Suppl 1): 3-11.

4. Cicardi M, Aberer W, Banerji A, et al. (2014): Classification, diagnosis, and approach to treatment for angioedema: consensus report from the Hereditary Angioedema International Working Group. Allergy 69: 602-616.

5. Gavigan G, Yang WH, Santucci S, et al. (2014): The prophylactic use of $\mathrm{C} 1$ inhibitor in hereditary angioedema patients undergoing invasive surgical procedures: a retrospective study. Allergy Asthma Clin Immunol 10: 17.

6. Keskindemirci G, Aktay Ayaz N, Aldemir E, et al. (2014): Familial mediterranean fever: diagnosing as early as 3 months of age. Case Rep Pediatr 2014: 296479. doi: $10.1155 / 2014 / 296479$.

7. Zuraw BL, Herschbach J (2000): Detection of C1 inhibitor mutations in patients with hereditary angioedema. J Allergy Clin Immunol 105: 541-546.

8. Serrano C, Guilarte M, Tella R, et al. (2008): Oestrogen-dependent hereditary angio-oedema with normal $\mathrm{C} 1$ inhibitor: description of six new cases and review of pathogenic mechanisms and treatment. Allergy 63: 735-741.

9. Weis M (2009): Clinical review of hereditary angioedema: diagnosis and management. Postgrad Med 121: 113-120.

10. Jacobs Z, Ciaccio CE (2010): Periodic fever syndromes. Curr Allergy Asthma Rep 10: 398-404.

11. Ghaffar TYA, Elsayed SM (2011): Colchicine resistant FMF is not always true resistance. Egypt J Med Human Genetics 12: 99-101.

12. Soriano A, Pras E (2014): Familial Mediterranean fever: Genetic Update. Isr Med Assoc J 16: 274-276. 